\title{
Operationalizing Flight Formations for Aerodynamic Benefits
}

\author{
Donald Erbschloe ${ }^{\mathrm{i}}$, Adam Antczak ${ }^{\mathrm{ii}}$, Dennis Carteriii, Gary Dale ${ }^{\mathrm{iv}}$, Carsten Döll ${ }^{\mathrm{v}}$, Robert Luckner ${ }^{\mathrm{vi}}$ \\ Tobias Marks ${ }^{\text {vii }}$, Michael Niestroy ${ }^{\text {viii }}$
}

\begin{abstract}
Nature abounds with examples of animals which decrease their energy expenditure by gliding off the bow waves of large moving objects, riding thermals, or flying in efficient configurations. The past twenty years has seen significant advances in the scientific understanding and feasibility of large aircraft saving energy by flying in advantageous pair or triple geometries. Several recent flight tests demonstrated reductions in fuel burn for trailing aircraft of $10 \%$ or more. The energy savings can be converted into a variety of beneficial operational capabilities: extended range, increased loiter time, larger payload, cost savings, etc. The next looming challenge is operationalizing this practice. The discussion areas include: benefits, penalties, and trade-offs; operational modes; maneuvers; routing and scheduling; regulations; and the process of a deliberate, evolutionary approach in fielding this capability into existing aviation enterprises.
\end{abstract}

\section{Introduction}

There are many factors which contribute to the feasibility of incorporating a new, potentially disruptive procedure such as efficient formation flight (or Air Wake Surfing for Efficiency-AWSE) into wellestablished and proven operational techniques and maneuvers. Basic operational challenges and opportunities include benefits from reduced fuel burn in the trailing aircraft, penalties incurred from pairing and tripling up aircraft, potential configurations, maneuvers, routing, scheduling, partnering, interoperability, authorities, permissions from air traffic control, and training.

\section{Fundamentals of AWSE}

\section{A. History}

Taking inspiration from nature is basic to powered flight —influencing shapes of wings, stabilizers, control surfaces, etc. In 1914 Carl Wieselsberger was the first who explained the energy advantage trailing birds in formation can exploit when flying in the upwash field created by leading bird ${ }^{1}$. Aerodynamic theory in this efficient maneuver was refined during the ensuing decades by Schlichting ${ }^{2}$ and $\mathrm{Hummel}^{3}$. Hummel was also instrumental, along with Beukenberg, at the Technical University of Braunschweig, to conduct flight tests in Dornier aircraft (starting in 1984) to validate not just the theory, but to validate in experiments and to initiate the development of automatic controls ${ }^{4}$.

\footnotetext{
${ }^{\text {i }}$ Consultant, Erbschloe Technical Consulting, Sheridan WY 82801; Senior member AIAA

ii Specialist, PLL LOT SA, Warsaw, Poland, 02-126

iii Retired, Xenia OH; Associate Fellow AIAA

iv Senior Research Engineer, Air Force Research Laboratory RQA, Wright-Patterson AFB, OH, 45433; Associate Fellow AIAA

${ }^{v}$ Research Scientist (Flight Control), ONERA, Toulouse, France

${ }^{v i}$ Professor, Technische Universität Berlin, Flight Mechanics, Flight Control and Aeroelasticity; Associate Fellow AIAA

vii German Aerospace Center, Hamburg, Germany 21079

viii LM Fellow, Control Sciences, Advanced Development Programs, Lockheed Martin Aero, AIAA; Associate Fellow
} 


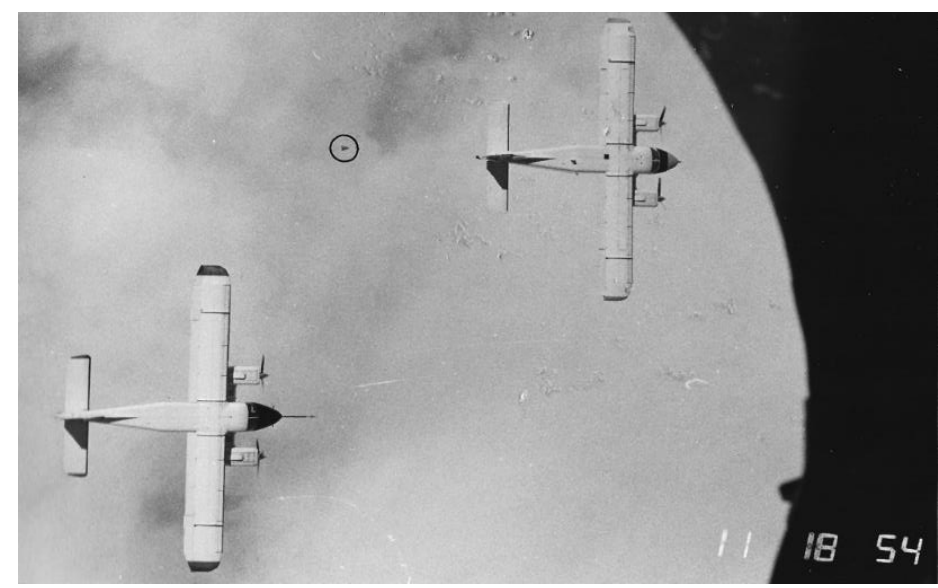

Figure 1: Overhead view of experimental test of formation theory using Dornier Do28 aircraft (1984)

Following the Braunschweig test flights, NASA conducted a series of efficient formation flight tests with fighters and transport aircraft ${ }^{6,7,8}$. A Defense Advanced Research Project Agency (DARPA) and Air Force Research Laboratory (AFRL) sponsorship, NASA teamed with Boeing to explore AWSE. Test flights in 2012 with this platform helped substantiate and quantify theoretical predictions and finetune automatic systems ${ }^{9}$. A significant flight test conducted in 2013 focused on maneuvers, procedures, human factors, safety, and compatibility with normal operations and air traffic control. NASA continued flight testing, most recently in the Gulfstream III, validating the use of Automatic Dependent Surveillance-Broadcast (ADS-B) as a datalink ${ }^{10}$. Commercial companies such as Lockheed-Martin and Airbus, academia, and government research laboratories (e.g., the Deutsches Zentrum für Luft- und Raumfahrt, DLR) have conducted extensive modeling and simulation and flights for AWSE. The flights demonstrated a fairly consistent fuel savings for the trail of 5-12\% for homogenous (same platform) and $18-30+\%$ savings for heterogeneous (e.g., a fighter aircraft following a large tanker or transport).

\section{B. The basics of aviation operational fuel efficiency}

Not surprisingly, interest in AWSE grows when jet fuel prices are high. Figure 2Error! Reference source not found. shows the past 3 decades of jet fuel prices. When there is a spike or high plateau in fuel costs, $\mathrm{R} \& \mathrm{D}$ funds follow. When the fuel returns to low values, funding (and customer interest) wanes.

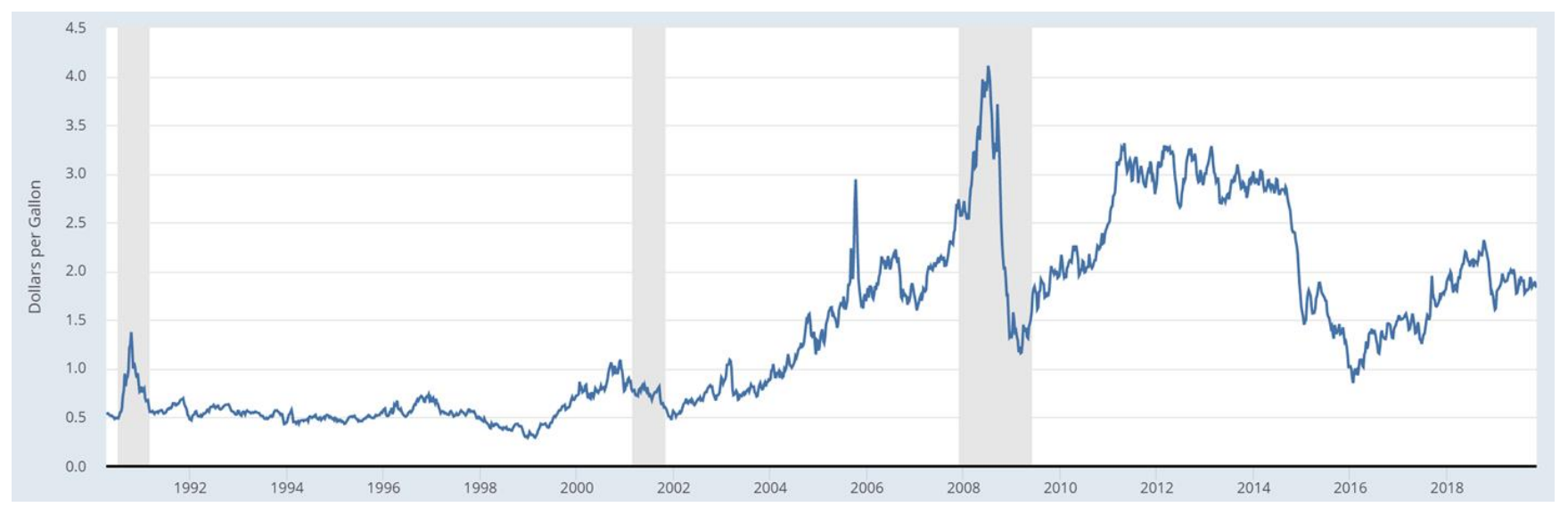

Figure 2: Jet Fuel Price (\$/gal) 1990-2019. Note: Arrows are periods with flight tests, grey bars represent recessions. ${ }^{11}$ 
Over the past two decades many studies have explored improving aviation system efficiency. ${ }^{12,13,14,15,16}$ In these studies, three broad approaches have been identified:

1. Boost the aircraft performance by increasing thrust specific fuel consumption (propulsion), improving Lift/Drag (aerodynamics), and/or reduce aircraft structural or payload weight

2. Improve the use of the aircraft through streamlined operations (e.g., advanced flight simulators, enroute modernization, best practices such as continuous descent approaches, etc.) ${ }^{17,18,19,20,21}$

3. Harvest energy from external sources (e.g., solar power, optimized use of favorable winds, and air wake surfing) ${ }^{22}$

\section{Decision making considerations: tradeoffs and penalties}

Whether or not to perform AWSE as a standard practice for a fleet requires a rigorous calculus, weighing benefits against penalties. According to Roberto I. Guerrero, Deputy Assistant Secretary of the Air Force for Operational Energy, "My office is interested in AWSE as an intriguing approach to tap into a resource of otherwise 'lost energy'. The energy gains have the potential for conversion into real operational benefits - extended range, longer loiter time, bigger payloads, or cost savings, to name a few.",23

1) Benefits

\section{Increased flight endurance}

Flight endurance is the maximum time an aircraft can stay airborne (at cruise). ${ }^{24}$ This is important in mission profiles which include loiter (cruising over a small area for an amount of time) such as reconnaissance, air refueling ovals, hunter/seeker, or command and control. The fuel saved by flying in AWSE can be used for greater time aloft.

\section{Extended range}

Fuel saved in efficient formation flight can be applied to fly longer distances. Models show this would be for missions like fighter drags, in which a large tanker aircraft ferries several smaller fighter aircraft long distances. The tanker generates a strong vortex with a magnified benefit on the smaller receiving aircraft.

\section{Additional payload}

Anticipated fuel savings could allow for the decrement of the fuel load prior to launch. The fuel weight saved could be substituted for payload.

\section{Fuel replenishment}

In special circumstances, such as humanitarian operations in disaster area, fuel is often limited at the destination airport. In this case, AWSE can save fuel, which reduces the requirement for onload for the return mission. Alternatively, the aircraft can leave excess fuel at the destination, replenishing the resources for further use.

\section{Tighter spacing}

The grouping of several aircraft for AWSE formations has the potential to increase the airspace capacity and reduce air traffic controller workload. This so-called "loose formation" can be beneficial to air traffic flow even if no aerodynamic benefit is achieved.

\section{Reduction of climate impact}

Fuel savings from AWSE can be part of a holistic approach for reducing greenhouse gases generated by aircraft (approximately $2 \%$ of human-produced $\mathrm{CO}_{2}$ annually). ${ }^{25}$ New standards and guidelines for emissions will impose restrictions and require technological and/or operational advances to meet the limits.

\section{Reduction in number and strength of vortices}

A two-ship formation generates four wingtip vortices. In AWSE, the inside vortices — one produced by lead and the other on the opposite wing by trail-are counter-rotating and in proximity. Although all four vortices interact dynamically, the two close vortices can react in a chaotic manner, but the expectation is an overall diminishment in strength, or even cancellation. 


\section{2) Penalties and inefficiencies}

\section{Suboptimal operating point}

Unless aircraft are perfectly matched in aircraft type and weight, they cannot both be flying at optimal conditions (e.g., speed and altitude). A compromise is required to maximize common benefits.

\section{Detours}

Aircraft originating from different airports need to fly to an agreed rendezvous point to create the AWSE formation. This generally adds distance to solo flight routes for one or both of the aircraft.

\section{Additional holdings}

If a rendezvous is necessary to pair up, precise timing is required to prevent one aircraft holding while awaiting the arrival of the other. This issue can be ameliorated to a degree if air traffic control allows for a flexible rendezvous point or the delay of the aircraft can be compensated prior to takeoff.

\section{Trim drag}

The flight within the wake vortex of another aircraft leads to additional aerodynamic drag induced by the compensation of the additional rolling moments (e.g. by aileron and rudder deflection) and must be included in the aerodynamic benefit model to properly predict the fuel savings. ${ }^{26,27}$

\section{Station Keeping Inaccuracy}

The benefit area in the (lateral/vertical) vortex plane is not symmetric about the optimum and drops off rapidly inside the optimum point. Significant induced rolling moments exist and station keeping systems have variance in position. The best strategy is to favor the outside of the optimum point. Furthermore, it can be assumed that the encounter of turbulence and gust during the flight will lead to a substantial loss of the benefits and therefore cannot be neglected. ${ }^{28}$

\section{Basic requirements to execute AWSE}

There are a handful of fundamental necessities to justify and be able to perform AWSE at the enterprise level. These are described next.

\section{A. Capable aircraft and systems}

To execute AWSE, an aircraft must position itself in the vortex updraft and maintain station. During the test flights in the 1980s and early 2000s, this task was relegated largely or entirely to the pilot. Many of the flights were flown in tight fingertip formation - with 1-2 wing spans separation-which some pilots called "white knuckle flights". Because the vortex persists for long distances behind the generating aircraft, the recommended method now is to maintain a large longitudinal separation (10-40 wingspans), greatly improving safety. A drawback is that the visual cues (e.g., parallax) that the trail depends on to maintain position are restricted at a distance. The aircraft must be able to predict or detect the location of the vortex, from which it must sustain a relative displacement. Automated systems, station-keeping equipment, datalinks, and proper displays are necessary.

\section{1) Automated systems}

Automated equipment in many military aircraft reduces pilot workload, keeps the aircraft on course, and maintains set headings, altitudes, and speeds. The automated systems and information which are required or desired to perform AWSE are:

- Autopilot: Lead (desired), Trail (required)

- Autothrottle: Lead (desired), Trail (highly desired)

- Measurement of winds aloft: Lead (required), Trail (required)

- Station-keeping equipment (SKE): Lead (required), Trail (required)

- Flight computer capable of AWSE calculations: Lead (desired), Trail (required) 


\section{2) Datalink}

Aircraft performing AWSE need to communicate and share vital information about each aircraft, intentions, and connectivity status. The respective AWSE element information to be sent is:

- $\quad$ Report position, velocity $(>2 \mathrm{~Hz})$

- Heading angle and warning if about to maneuver

- Meteorological message, including winds (desired)

- Weight (occasionally)

- Acknowledgement of information received (desired)

Available datalinks are incorporated in dedicated flight formation systems and SKE (e.g., Leonardo AN/AP-243 Station Keeping Equipment ${ }^{29}$ and Honeywell MILACAS-FR Formation-flying systems ${ }^{30}$ ). However, more affordable solutions exist-NASA Armstrong recently completed a successful test of transmitting and receiving adequate information using ADS-B out (a mandatory capability for aircraft transiting Mode C transponder airspace by 2020). ${ }^{31}$

\section{3) Sensors/Instrumentation}

Physical models can predict the location and strength of the vortex, however direct measurement using systems like Doppler LIDAR or radiometers would be very useful. Standard cockpit instrumentation can display behaviour indicative of aerodynamic benefit and proximity to the vortex: fuel flow and angle of attack should decrease while aileron trim should increase. Such cues could be used for peak seeking and optimization. Sensors placed along the wing can help pinpoint the wake position. ${ }^{32}$

\section{4) Displays}

Pilots executing AWSE want good situational awareness. They want to know their position relative to the vortex (and where they can and should not fly) and some sense of fuel benefits they are accruing. Researchers at the Technical University of Berlin designed a notional display (see Error! Reference source not found.) for a cockpit monitor or could be an app on an Electronic Flight Bag. ${ }^{33}$

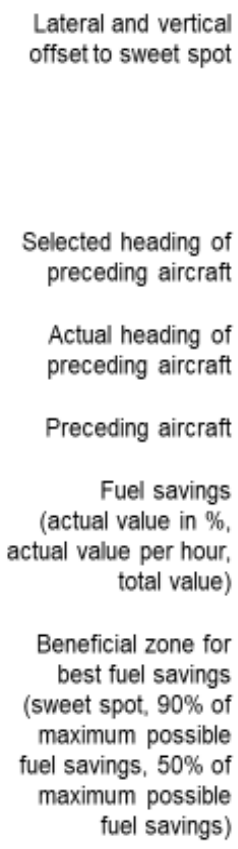

Lateral and vertical offset to sweet spot

Selected altitude of preceding aircraft

Actual altitude of preceding aircraft

Danger zone, keep out own CoG at any time

(transparent orange zone: wingtips are allowed but pilots should be aware that the wings are placed in the vortex core: transparent red zone: keep out own wingtips at any time)

Longitudinal distance to preceding aircraft

Ovn aircraft (CoG, wings)

Figure 3: Notional Display for AWSE 


\section{B. Trained and qualified aircrews}

A source for trained and qualified aircrews is the military. Military pilots undergo comprehensive and rigorous and training across a broad spectrum of procedures, and techniques. They also must be qualified and certified for specific maneuvers.

Boeing and AFRL developed procedures and aircrew training modules for employment of AWSE. Several instructor, examiner, and test pilots were involved in the development and demonstration phases. The comments from this group provided useful insight and suggestions, including that airwake surfing procedures would be easy to train in the simulator. A current and proficient tactical formation qualified pilot could be certified with a single simulator flight, while non-formation qualified pilots might require one simulator and one flight.

Commercial pilot training and certification would be more extensive, although much of that could be accomplished in a simulator. Bieniawski, et al., ${ }^{34}$ and Blake ${ }^{35}$ confirmed that significant fuel savings for airline fleets in general using AWSE. ${ }^{36}$. A Stanford study ${ }^{37}$ demonstrated for real air traffic networks the significant potential fuel saving. It was maximal if airplanes of different airlines that fly along the same flight corridor would fly in formation, e.g. a fuel saving of $6.8 \%$ would be feasible for the transatlantic network of the Star Alliance.

\section{Operational compatibility}

AWSE operations must conform to normal air operations to be accepted and adopted by fleets. Simply put, this is a new set of maneuvers, but they should not be unfamiliar. Challenges to implementing the practice of AWSE must be considered and overcome.

\section{1) Longitudinal Displacement}

A vortex is typically fully formed and stable typically within 1.5 wingspans downstream from the generating aircraft and begins to break up after a few minutes - several miles--behind the aircraft. The trail aircraft therefore needs to be placed in the stable part of the vortex (test flights were usually flown. 30006000 feet behind lead). Other constraints influencing the longitudinal displacement include those imposed by Air Traffic Control.

\section{2) Lateral displacement}

A distinguishing feature of AWSE is that the trailing aircraft must position itself relative to the vortex core rather than relative to the lead aircraft (see Figure 4). Due to the nonuniform inflow on the followers wing an additional rolling moment is induced that needs to be compensated for by aileron and rudder deflections. The magnitude of the rolling moment depends on the lateral and vertical displacement. Areas where the rolling moment exceeds the compensation capabilities of the trail or where a change of sign occurs need to be avoided.

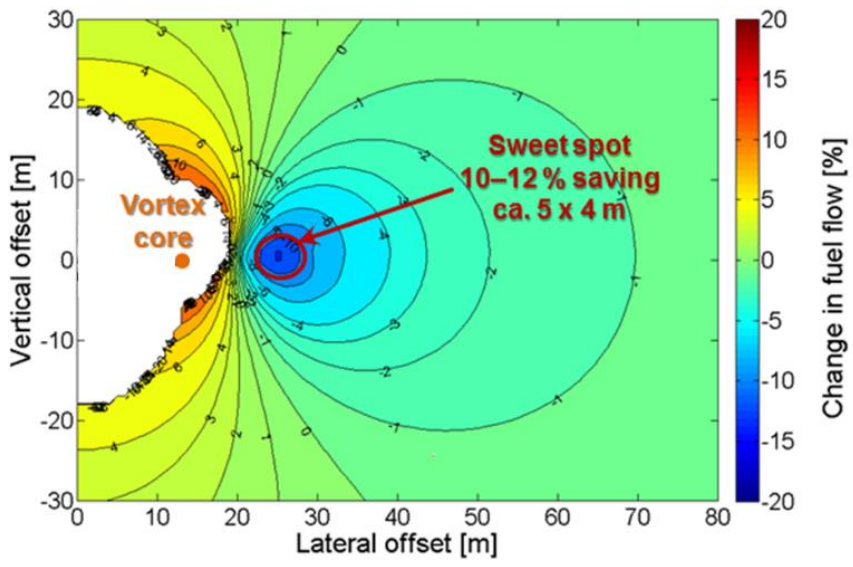

Figure 4: Fuel savings benefit map off vortex $\operatorname{core}^{38}$

The desired lateral displacement will also depend on the flight conditions. At night or in IMC (Instrumented Meteorological Conditions), the crew in the trailing aircraft may decide to fly a loose geometry with greater 
separation from the vortex. Automated formation systems are IFR (Instrument Flight Rules) certified; without such systems, AWSE may be limited to VMC (Visual Meteorological Conditions).

Crosswinds will push the vortex away from the earth-referenced flight path of the lead aircraft. In order to compensate for these effects, detailed models of the vortex movement and an exchange of meteorological data between the aircraft are necessary.

\section{3) Vertical displacement}

The lead aircraft generates two counter-rotating vortices. These do not act independently, rather they form a dynamic system where each vortex pushes down the other. The result is vortices which descend in time. The greater the longitudinal distance behind lead the drop increases. The descent rate is modest, for example the vortex descent behind a medium size transport at cruise is roughly $7 \mathrm{ft} / \mathrm{s}$. Flying at M0.75, the expected descent of the vortex is roughly 50 feet at one nautical mile in trail. Since the recommended longitudinal separation is a few thousand feet to $2 \mathrm{~nm}$, the trail aircraft will still be within 100 feet of lead vertically. This is important for Air Traffic Control, who must keep aircraft separated by safe vertical distances.

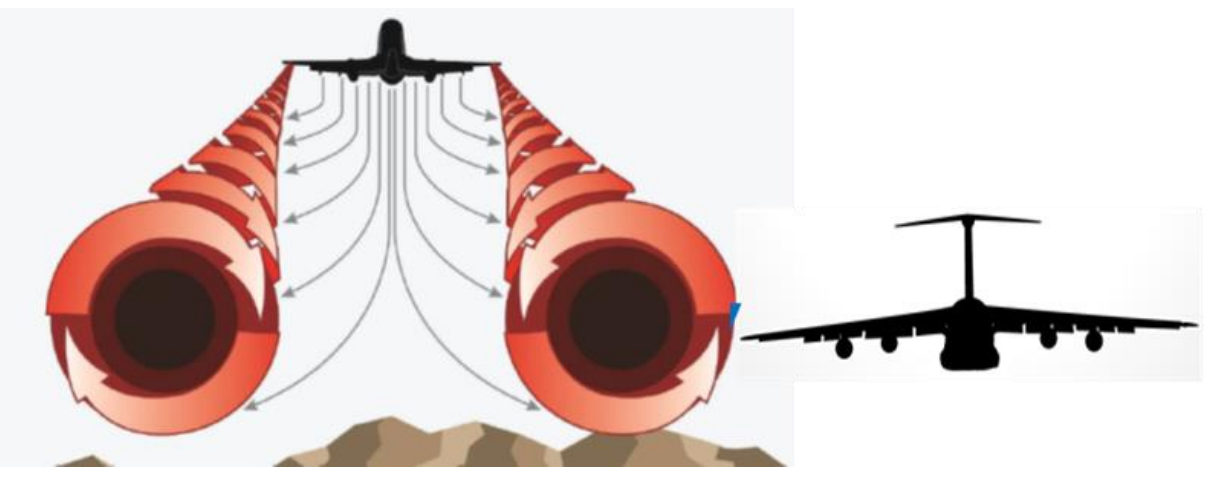

Figure 5: Counter-rotating vortices and vertical displacement of trail ${ }^{39}$

\section{4) Configurations}

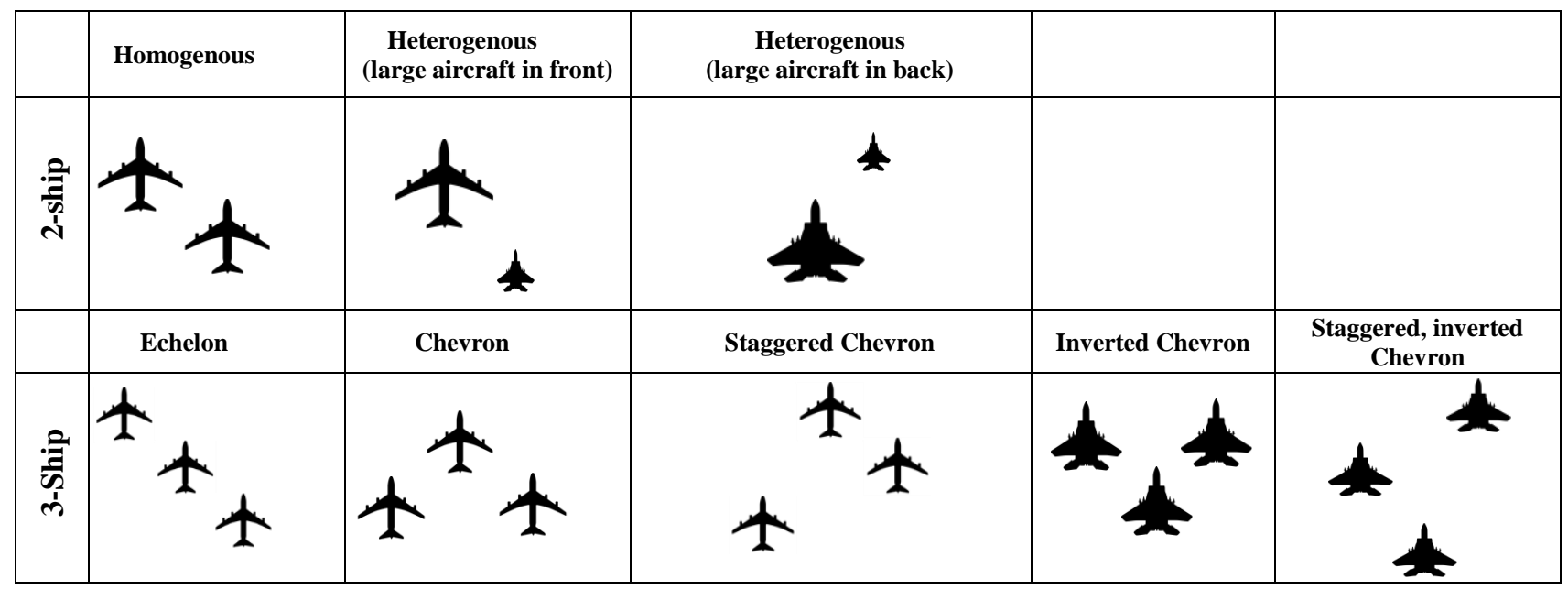

Table 1: Formation Configurations ${ }^{40}$

A fundamental question concerning the operation of aircraft in formation is the number of participants as it influences the technical systems as well as the operational procedures. Formations with large number of aircraft are more difficult to maintain and control but yield the highest benefits. Vortices may overlap, cancel themselves or behave in a chaotic way. Small formations are easy to establish and control. 
Depending on the number and types of aircraft different arrangements are possible (Table 1: Formation Configurations). Operators should look at missions in which aircraft are sufficiently close proximity for extended periods of non-maneuvering flight and exploit those first.

5) Airport usage

Another important operational question that needs to be defined is whether the participants take off from the same airports or join together from different airports. Using the same airport can maximize the benefits as the aircraft can fly together as long as possible. Using different airports yields more opportunities for pairing up aircraft but can lead to significant detours that reduce the benefits.

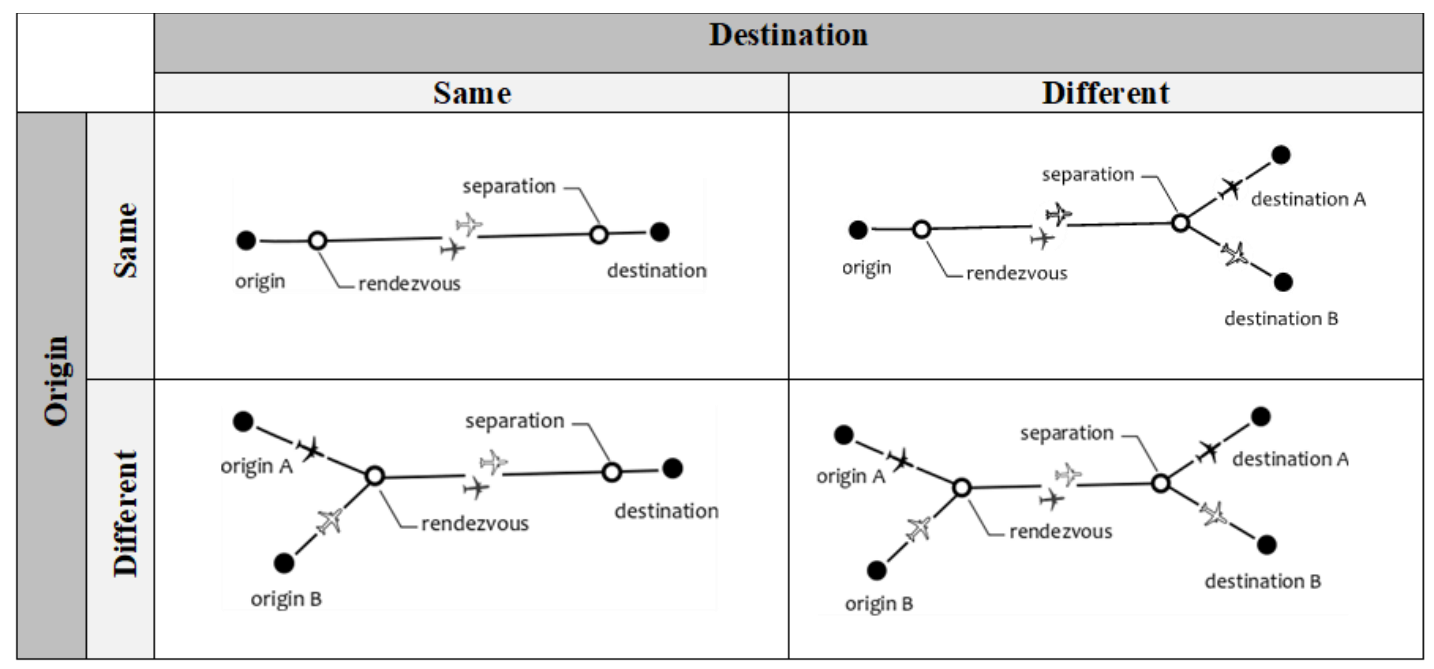

Table 2: Different operational scenarios for formation flight ${ }^{41}$

Table 2 shows the various possibilities arising from different departure and arrival airports. Pros and cons of the different options are:

\section{Same-Different}

Departing at the same airport reduces the coordination of the formation members significantly. In this case a sequential departure can be conducted with one formation member performing a holding pattern or flying at speeds which allow a smooth join to the formation. .

\section{Different-Same}

Arriving at the same destinations yields similar operational problems as in the case of same origins, as after the separation the formation members need to land sequentially if no independent runways are available. This requires additional coordination among the formation members.

\section{Same-Same}

The same origin and destination of the formation members yields the highest benefits in terms of fuel savings as the length of the formation route can be maximized. However, the take-off and landing procedure needs to be adapted if no independent runways are available at the origin and destination airport.

\section{Different-Different}

This case allows the highest flexibility in joining up the aircraft, as a formation can be established among all suitable flights.

\section{6) Maneuvers}

A recommendation of this article is to initially limit the application of AWSE to extended, non-maneuvering phases of flight. In Figure 6, this would be during cruise and possibly the region in climb or descent close 
to level flight. As confidence in AWSE grows, more of the climb and descent might be included, however efficient formation flight in the takeoff, departure, approach (instrument or visual), and landing phases will likely remain forbidden.

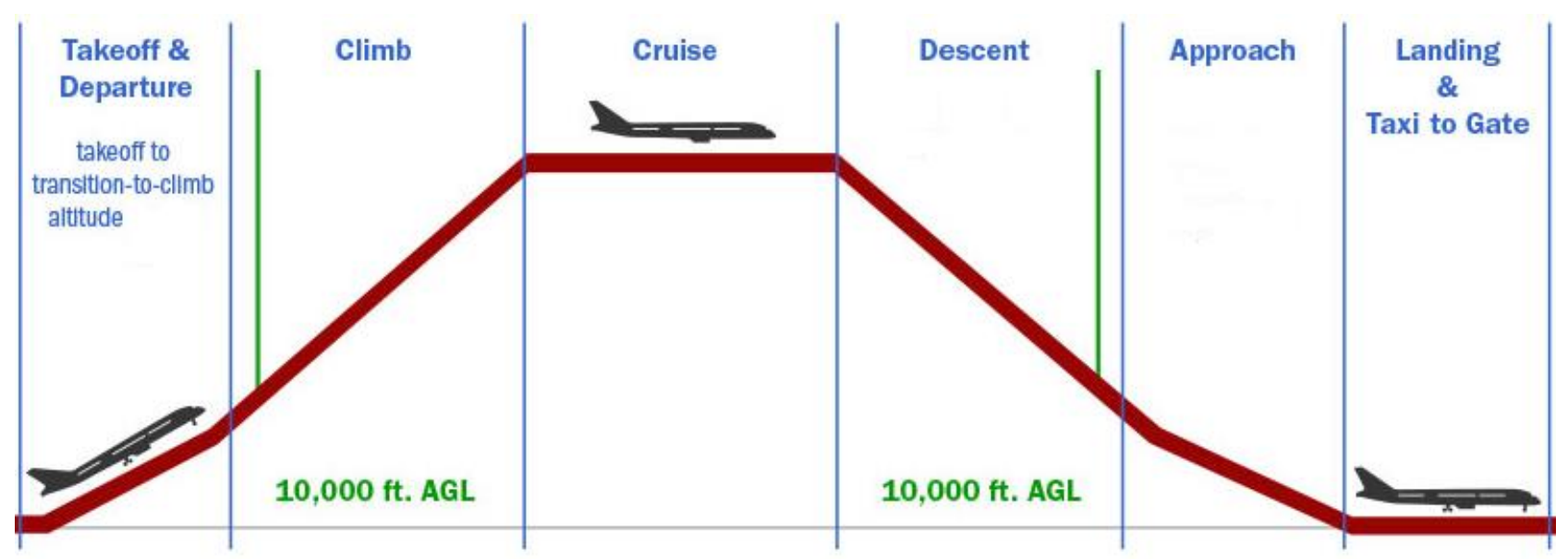

Figure 6: Phases of flight ${ }^{42}$

Additional useful maneuvers are described below.

\section{Positional changes}

Positional changes can be observed in the formation flight of birds. It occurs if the leading bird gets tired, falls back, and is replaced by a fresh flock member. In powered flight with similar platforms, the trailing aircraft burns less fuel than the leader. It is reasonable to consider a change of positions during the formation in order to share benefits. Such a decision must balance the savings against the cost of aircrew training and currency to perform this maneuver.

\section{Course adaptations}

Minor course adaptations should be permissible while in formation. If larger course adaptations need to be performed the formation needs to break up and rejoin after the adaptation is finished. In order to synchronize the movement, all aircraft in the formation need a stable datalink.

\section{Separation}

It will be routine for the trailing aircraft in AWSE to separate either for short periods of time (e.g., when it is necessary for one of the aircraft to perform some necessary flight task or when forecast or reported weather issues, such as IFR conditions or increased turbulence) or permanently (e.g., when the formation leg of a different destination AWSE is complete or in failure cases). In either case, the trailing aircraft should announce the separation to lead and move to a safe lateral displacement (250-500 feet is adequate and gets the trail into a position where the updraft is minimal). If the separation is temporary, trail should request permission to reassume AWSE position and receive confirmation from lead before moving back.

\section{Breakaway}

A breakaway can be called by any aircraft in the formation when a safety of flight issue arises (e.g. unexpected moderate or greater turbulence, unintentional wake/vortex crossing, failure conditions, etc.). An immediate separation should be initiated. A rejoin is possible after the causal condition is mediated.

\section{Novel maneuvers}

As AWSE is a new, albeit familiar, way of flying we anticipate that aircrews will discover new, unforeseen procedures and maneuvers to improve safety and effectiveness. Two novel maneuver examples which have been previously discussed are presented to spur innovative juices. 
Upwash cradle:

A 1976 paper by Donaldson proposed an intriguing configuration, which is almost the inverse of a staggered V formation ${ }^{43}$. As shown in Figure 7, the outermost aircraft assume lead positions, in a staggered manner (although for safety, the recommendation is to have the leads longitudinally displaced by 1000-2000 feet). In this configuration the trail benefits from the upwash of both lead aircraft. With precise placement, the superposition of these two could result in an induced thrust (vice an induced drag) for the trailing aircraft.

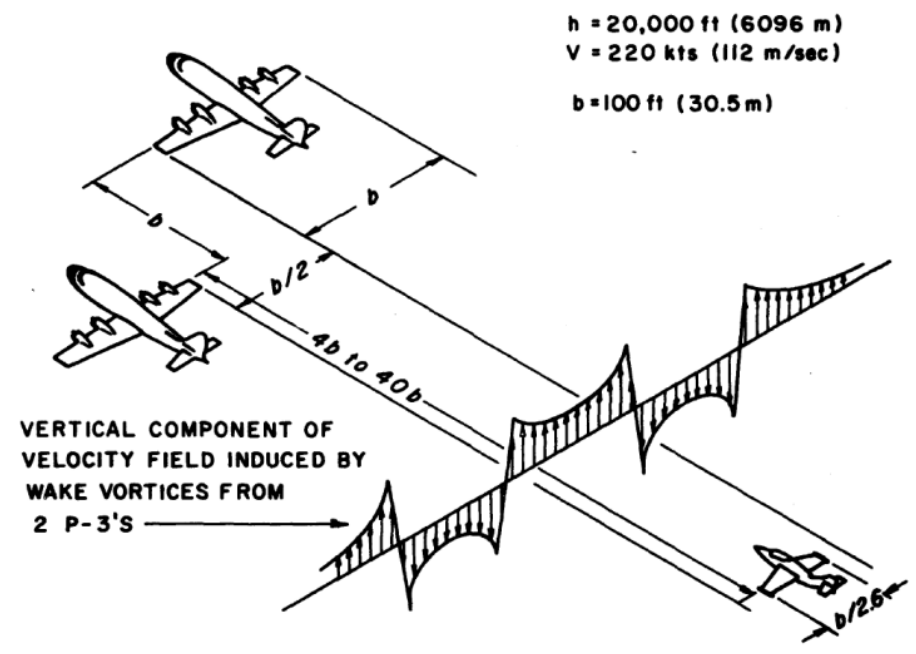

Figure 7: Configuration for upwash cradle - the rearmost aircraft flies in the upwash of each of the two aircraft ahead (from Reference 43)

Leapfrogging:

Leapfrog is a game in which one person uses the back of a hunched over leader to propel himself or herself ahead of lead. In aviation, a similar boost can be achieved when a trailing aircraft joins up on a lead, receiving a fuel savings benefit, and can then reach a destination which would have been unavailable to it flying solo.

\section{7) Routing / Operating point}

Flight paths can be altered due to constraints given by air traffic control, mission requirements (including avoiding threat areas) or optimization (e.g. favorable winds or more direct routing). In order to realize the maximum absolute fuel savings in a formation, it is essential to optimize the routes of the participating members and the group altogether. Optimization also includes the locations of the rendezvous and separation points; the formation track as well as the altitude and speed of the formation.

The need for optimization is strongly defined by the selected CONOPS. Airport usage determines whether the rendezvous or separation points are subject to optimization. The highest benefits can be expected if the formation members depart from the same airports and go to the same destination. If the loading and aircraft types are identical the formation can fly at an optimal operating point.

\section{Mission type}

Depending of the type of mission that needs to be accomplished (e. g. surveillance, cargo transfer, fighter drag) different optimization criteria apply. For example, an optimal operating point and formation track will differ if a maximum flight time or a cost saving should be realized. 


\section{Optimization of rendezvous and separation points}

The geographic location of the rendezvous and separation points strongly influences the benefits depending on the airport scenarios. Detours lead to a decreased benefit; however, one formation member may need a large detour in order to join an optimized formation. A seasonal variation in winds can affect the location of optimal rendezvous and separation points. Fixed rendezvous and separation points can lead to a decrease in efficiency that can reach up to $1 \%$ of total burned fuel. ${ }^{44}$

\section{Optimization of operating point}

The compromise to fly at the same altitude and at the same speed necessitates the optimization of both factors in order to achieve the highest benefits for the whole formation. Simultaneous step climbs can be an option to achieve even higher benefits.

\section{Daily changes}

Daily changes like wind patterns strongly influence the optimization and can lead to a decrease in benefit. In order to simplify the strategic optimization (e.g. fuel planning) statistical information about typical wind situations can be used. For the tactical planning weather forecast data needs to be considered to determine the optimal flight tracks.

\section{Flight plan management}

Airlines in order to create flight plan for every operation are using dedicated systems (e.g. Netline, Sabre). Those software systems are designed to build the flight plan for every known conditions (e.g. weather, tracks, aircraft limits, number of passengers) and business rules (fastest, cheapest etc.). If the formation flight module would be available, all the changes and optimization would be done here.

\section{8) Scheduling/Partner Allocation}

Some operators may hesitate to adopt AWSE from a perception that pairing is too difficult to manage in a large enterprise or that the benefit generated from a low number of potential pairs does not make a compelling business case. An initial glance at a daily flight schedule seems to suggest this. This is by design. The historic scheduling process purposely deconflicts most aircraft flying similar routes at similar times. For example, positioning/depositioning aircraft transporting equipment and/or troops for an exercise is typically executed by staggering takeoff/landing times.

Two modifications to building schedules and matching aircraft dramatically increases the number of potential pairs: 1) allow flexibility in takeoff times and 2) pair up aircraft taking off and/or landing at different airfields if the fuel savings outweigh the cost of rendezvous and route change. Boeing analyzed a year's worth of mobility transport flights in the North American region, allowing for pairing of C-17 aircraft originating and/or terminating at different airports (up to 1,000 nm apart) and up to a 12 hour shift in takeoff time. ${ }^{45}$ Allowing a 1-hour shift in the scheduled departure times produced a 6 -fold increase in possible pairs over the number with an inflexible schedule. Even a modest 2-hour shift in take-off time would have achieved 2/3s of the maximum benefit. A 4-hour shift results in $90 \%$ of the maximum benefit.

If the operator can generate, and manage the flying schedule, it can be creative. In the exercise positioning/depositioning scenario, pairing up adjacent launches could result in fuel savings and, potentially, allow for quicker closure. Another target is a schedule in which there are daily flights between airbases. A more efficient approach would be to pair up aircraft every other day. The (near-) simultaneous take off/landing should not overwhelm ground support.

Originating aircraft from different bases presents a complication. If this is not coordinated well or there is a delay and one aircraft has to hold at the rendezvous point, then any projected fuel savings will rapidly dwindle away. This can be ameliorated by adopting commercial practices (i.e., coordinating engine starts), adjusting relative speeds inflight to achieve the best closure and creating a dynamic rendezvous calculator that builds in time delays. 


\section{9) Proper authorities}

Authorities and allowances for joining up of aircraft already exist in guidance and directive documents from regulatory agencies for aviation. Relevant language on formation flights include:

FAA JO 7110.65W, Air Traffic Control ${ }^{46}$

Formation flights are treated and controlled as a single aircraft and will continue until separation is established. Separation procedures are detailed.

FAA Part 91 Sec. 91.111 Operating near other aircraft ${ }^{47}$

Pilots in command of all aircraft in a formation must agree to the formation and coordinate actions and roles.

ICAO Annex 2 Sec. 3.1.8, Rules of the Air ${ }^{48}$

ICAO rules are similar to the FAA rules. These add that formation coordination extends to periods of transition (e.g., join up and separation). In addition, separations to not exceed $1 \mathrm{~km}$ laterally and longitudinally and $30 \mathrm{~m}$ vertically from flight lead are established.

\section{Target applications (near-term, mid-term, far-term)}

AWSE is being pursued by several different research groups (e.g., DLR, NASA, AFRL, etc.), operators and aircraft companies (e.g., Airbus, Boeing, Lockheed-Martin, etc.). The applications vary from nearterm (can be adopted in less than 5 years), the mid-term (5-10 years), or far-term (greater than 10 years). Three representative use-cases, each with a unique perspective on the employment of efficient formation flight are suggested. These cases can start with rigorous modeling \& simulation and wargaming and culminate in operational flight tests.

A. Near-term: Homogeneous mix. Rigorous modeling \& simulation and wargaming, looking at a variety of similar platform pairs. These should culminate in operational flight tests.

B. Mid-term: Heterogeneous mix. A fighter aircraft in formation with a large aircraft (normally an air refueling tanker) is useful construct. Indeed, roughly 1 in 20 sorties for USAF air refueling aircraft involves the movement (or "ferry") of fighter aircraft long distances (CORONET missions). The USAF has looked at the formation flight efficiencies in CORONETs and one study determined that incorporating AWSE-like arrangements could allow the tankers to increase the number of fighters carried, or, alternatively, reduce the number of required tankers by one (Reference 43).

C. Far-term: Autonomous aircraft (paired with other autonomous or human-crewed aircraft). Development in both autonomy and understanding of a heterogeneous mix must be accomplished before this is considered feasible. However, an autonomous aircraft offers multiple advantages as a partner. Many of the challenges facing the adoption of AWSE relate directly to aircrews or passengers, such as training/currency requirements and ride quality issues. These issues disappear if the receiving aircraft is unoccupied. Military examples include autonomous aerial refuelling ${ }^{49}$, aircraft companions ${ }^{50}$, drones, and swarms of drones. A common theme of these military programs is that the requirements and systems to make these work (e.g., automated controls, datalinks) will also enable AWSE. As imagined in the aircraft companion program, the synergy of a piloted lead aircraft with autonomous companion(s) is compelling. In many scenarios the robotic partner is a smaller aircraft carrying additional sensors, communications equipment, armaments, etc. As NASA observed in the combination of fighters and transport aircraft, the expectation is that the smaller, lighter aircraft could see fuel savings on the order of $10-20 \%$. The fuel savings can be traded for operational benefit: extended range, longer loiter time, additional payload, etc. 


\section{Conclusions}

Introducing and adopting AWSE as a standard practice will initially be disruptive and there will likely be some scepticism and resistance. This is understandable and suggests a gradual accession into operations. Two related guiding principles can help:

- Start with use of efficient formation flight where and when aircraft fly for extended periods of time in close proximity - for example, tactical formations enroute or returning from areas of operation; positioning or depositioning of aircraft and equipment for exercises; tanker cells (air refuelers often pair up enroute to/from orbits); fighter drags (when multiple fighter aircraft are ferried long distances with one or two air refuelers - known as CORONET missions in the USAF).

- Limit efficient formation flight initially to non-maneuvering phases of flight.

Minimizing energy expenditure is a common practice in nature: dolphins (and human surfers) ride the bow waves off ships, hawks (and gliders) circle in thermals to gain altitude and potential energy, and birds like geese fly in v-shaped formations to reduce their exertions during long migrations. It was inevitable when humans took to the skies in the early days of powered flight to take cues from nature. There have been many attempts over the decades to fly in formations, like birds, for energy efficiency. These trials demonstrated fuel savings - often an impressive amount — but came with a huge workload cost. In recent times, improved automation, station keeping, ability to pass data between aircraft, and enhanced tools for situational awareness make a procedure like AWSE possible and practical. The fuel saved by a trailing aircraft can be traded for a variety of operational benefits: increased range, longer endurance, increased payload, cost savings, and reduction in emissions and carbon footprint. This is true for military as well as civil airline operations.

\footnotetext{
${ }^{1}$ Wieselsberger, C., "Beitrag zur Erklärung des Winkelfluges einiger Zugvögel”. Zeitschrift für Flugtechnik und Motorluftschiffahrt 5, 1914.

${ }^{2}$ Schlichting, H., "Leistungsersparnis im Verbandsflug". Mitt. d. Deutsch. Akad. d. Luftfahrtforschung., Heft 2: 97

- 139. Excerpts in: Jahrbuch 1942/43 d. Deutsch. Akad. d. Luftfahrtforschung., 1942.

${ }^{3}$ Hummel, D., "Aerodynamic aspects of formation flight in birds". Journal of Theoretical Biology 104, 1983.

${ }^{4}$ Beukenberg, M. \& Hummel, D., "Aerodynamics, Performance and Control of Airplanes in Formation Flight". Proceedings of the 17th Congress of the International Council of the Aerospace Sciences 1990 Stockholm, Vol. 2, 1990.

${ }^{5}$ Photo courtesy of Technical University Braunschweig

${ }^{6}$ Morgan, Michael, T., "A Study in Drag Reduction of Close Formation Flight Accounting for Flight Control Trim Positions and Dissimilar Formations," Thesis, Air Force Institute of Technology, 2005. Available online at: https://apps.dtic.mil/docs/citations/ADA434312

${ }^{7}$ Wagner, G., Jacques, D., Blake, W., and Pachter, M., "Flight Test Results of Close Formation Flight for Fuel Savings," AIAA-2002-4490, Proceedings of the AIAA Atmospheric Flight Mechanics Conference and Exhibit, Monterey, California, 2002. Available online at: https://doi.org/10.2514/6.2002-4490

${ }^{8}$ Anonymous, "NASA Sky Surfing for Fuel Economy," NASA press release, July 21, 2003. Available online at: https://www.nasa.gov/missions/research/vortex.html

${ }^{9}$ Bienawski, S.R., Clark, R.W., Rosenzweig, S.E., and Blake, W.B., "Summary of Flight Testing and Results for the Formation Flight for Aerodynamic Benefit Program," AIAA 2014-1457, Presented at the 52 ${ }^{\text {nd }}$ Aerospace Sciences Meeting, AIAA SciTech, 2014. Available online at: https://doi.org/10.2514/6.2014-1457

${ }^{10}$ Hanson, C., Pahle, J., Reynolds, J., Andrade, S., and Brown, N., "Experimental Measurements of Fuel Savings During Aircraft Wake Surfing," AIAA 2018-3560, Presented at the AIAA Atmospheric Flight Mechanics Conference, Atlanta, Georgia, June 25-29, 2018

${ }^{11}$ Available online at: https://fred.stlouisfed.org/series/WJFUELUSGULF\#0

${ }^{12}$ AF Scientific Advisory Board, Technology Options for Improved Air Vehicle Fuel Efficiency, SAB-TR-06-04, May 2006.

${ }^{13}$ National Research Council, Air Force Studies Board, Improving the Efficiency of Engines for Large Nonfighter Aircraft, 2007. Available online at: https://www.nap.edu/catalog/11837/improving-the-efficiency-of-engines-forlarge-nonfighter-aircraft.
} 
${ }^{14}$ Defense Science Board, DoD Energy Strategy, "More Fight, Less Fuel”, 2008. Available online at: https://www.idga.org/energy-power-savings/whitepapers/more-fight-less-fuel-infographic

${ }^{15}$ Airbus, The Future, 2010. Available online at: https://www.airbus.com/newsroom/pressreleases/en/2010/07/airbus-concept-plane-offers-glimpse-into-the-future-of-flight.html

${ }^{16}$ AF/ST TR 11-01, Energy Horizons, Jan 2012. Available online at: https://www.hsdl.org/?view\&did=741379

${ }^{17}$ Government Business Council, Going Virtual to Prepare for a New Era of Defense, 2015. Available online at: https://www.govexec.com/gbc/going_virtual_for_new_defense_era/

${ }^{18}$ Available online at: https://www.faa.gov/air_traffic/technology/eram/.

${ }^{19}$ FAA Advisory Circular 120-108, Continuous Descent Final Approach, Jan 20, 2011. Available online at: https://www.faa.gov/regulations_policies/advisory_circulars/index.cfm/go/document.information/documentID/8773 39

${ }^{20}$ Boeing, Fueling Efficiency, in Boeing Frontiers, Nov 2010. Available online at: http://www.boeing.com/news/frontiers/archive/2010/november/

${ }^{21}$ Available online at: http://www.geaviation.com/commercial/services/flight-efficiency-services/ [cited 10 January 2016]

${ }^{22}$ Available online at: http://www.digitaljournal.com/science/solar-impulse-2-the-amazing-technology-behind-theflight/article/427899 [cited 18 August 2015]

${ }^{23}$ Quote courtesy of Roberto Guerrero

${ }^{24} \mathrm{https} / / /$ en.wikipedia.org/wiki/Endurance

${ }^{25}$ Intergovernmental Panel on Climate Change (IPCC), "Aviation and the Global Atmosphere", 1999. Available online at: https://www.ipcc.ch/report/aviation-and-the-global-atmosphere-2/

${ }^{26}$ Okolo, W., Dogan, A., and Blake, W., "Application of Sweet Spot Determination to a Conventional Pair of Aircraft," AIAA Atmospheric Flight Mechanics Conference, AIAA 2012-4402, Minneapolis, Minnesota, 13-16 August 2012. doi: 10.2514/6.2012-4402

${ }^{27}$ Kaden, A., and Luckner, R.: "Modeling Wake Vortex Roll-Up and Vortex-Induced Forces and Moments for Tight Formation Flight," AIAA Modeling and Simulation Technologies Conference and Exhibit, 2013. doi: 10.2514/6.20135076

${ }^{28}$ Marks, T., Zumegen, C., Gollnick and V., Stumpf, E. “Assessing formation flight benefits on trajectory level including turbulence and gust", AIDAA Italian Association of Aeronautics and Astronautics, XXV International Congress, 2019. Available online at:

https://www.researchgate.net/publication/336798728_Assessing_formation_flight_benefits_on_trajectory_level_incl uding_turbulence_and_gust

${ }^{29}$ Available online at: https://www.leonardodrs.com/products-and-services/anapn-243-stationkeeping-equipment2000-ske-2000/

${ }^{30}$ Available online at: https://dokumen.tips/documents/milacas-fr-formation-flying-system-honeywell-ensures-thatthe-warfighter-has-the.html

${ }^{31}$ Available online at: https://www.aopa.org/advocacy/advocacy-briefs/air-traffic-services-brief-automaticdependent-surveillance-broadcast-ads-b

${ }^{32}$ Fischenberg, D. “Online Wake Identification Algorithms Using Forward Looking LIDAR Sensor

Measurements", German Aerospace Center, IB111-2013/11, 2013. Available online at:

https://www.semanticscholar.org/paper/In-flight-remote-sensing-and-identification-of-and-Fezans-

Schwithal/39fe168235960d4d7ce2f1226baf9bcf397e34f0.

${ }^{33}$ Kaden, A., and Luckner, R.: "Maneuvers during Automatic Formation Flight of Transport Aircraft for Fuel Savings," AIAA SciTech, Orlando, 6-10 January 2020, submitted for publication.

${ }^{34}$ S. R. Bieniawski, S. Rosenzweig und W. B. Blake, "Summary of Flight Testing and Results for the Formation Flight for Aerodynamic Benefit Program," AIAA SciTech, 52nd Aerospace Sciences Meeting, 2014.

${ }^{35}$ W. B. Blake, S. R. Bieniawski und T. C. Flanzer, "Surfing Aircraft Vortices for Energy," The Journal of Defense Modeling and Simulation, Bd. 12, Nr. 1, p. 31-39, 2015. Available online at: https://doi.org/10.2514/6.2014-1457

${ }^{36}$ J. Pahle, D. Berger, M. Venti, C. Duggan, J. Faber und K. Cardinal, "An Initial Flight Investigation of Formation Flight for Drag Reduction on the C-17 Aircraft,“ 2012. Available online at: https://doi.org/10.2514/6.2012-4802

${ }^{37}$ J. Xu, A. Ning, G. Bower und I. Kroo, "Aircraft Route Optimization for Formation Flight," Journal of Aircraft, Bd. 51, Nr. 2, p. 490-501, 2014. Available online at: https://doi.org/10.2514/1.C032154

${ }^{38}$ Koloschin, A., and Vechtel, D., "Towards Automatic Control of Fuel-Saving Formation Flight," German Aerospace Congress, 2018. Available online at: https://elib.dlr.de/122523/ 
${ }^{39}$ FAA, Advisory Circular 90-23G, Aircraft Wake Turbulence, pg 2, Available online at: https://www.faa.gov/documentLibrary/media/Advisory_Circular/AC_90-23G.pdf.

${ }^{40}$ D. Erbschloe, AVT-279 original work product, Formation Configurations

${ }^{41}$ D. Erbschloe and T. Marks, AVT-279 original work product, Different operational scenarios for formation flight

${ }^{42}$ Available online at: https://aviation.stackexchange.com/questions/29460/what-is-a-hypernym-for-the-ascendingand-descending-legs-of-a-flight

${ }^{43}$ Donaldson, Alan J., et al., "Study of the Feasibility of Conducting a Wake-Riding Experiment using a T-2 aircraft behind two P-3 aircraft", Naval Air Development Center (1976). Available online at:

https://apps.dtic.mil/dtic/tr/fulltext/u2/a033101.pdf

${ }^{44}$ Marks, T., Swaid, M., Lührs, B. and Gollnick, V. "Identification of optimal rendezvous and separation areas for formation flight under consideration of wind", $31^{\text {st }}$ Congress of the International Council of the Aeronautical Sciences (ICAS) (2018)

${ }^{45}$ T. C. Flanzer, S. R. Bieniawski, and W. B. Blake. "Operational Analysis for the Formation Flight for Aerodynamic Benefit Program”. In: AIAA SciTech 52nd Aerospace Science Meeting. AIAA 2014-1460. National Harbour, Maryland, 2014. doi: 10.2514/6.2014-1460.

${ }^{46}$ FAA Order JO 7110.65W Air Traffic Control, pg 2-1-6, Available online at: https://www.faa.gov/documentlibrary/media/order/atc.pdf

${ }^{47}$ Available online at: https://www.law.cornell.edu/cfr/text/14/91.111

${ }^{48}$ ICAO, Rules of the Air, pg 3-1, Available online at: https://www.icao.int/Meetings/anconf12/Document\%20Archive/an02_cons\%5B1\%5D.pdf

${ }^{49}$ Available online at: https://news.northropgrumman.com/news/releases/photo-release-X-47b-unmanned-aircraftdemonstrates-the-first-autonomous-aerial-refueling

${ }^{50}$ Available online at: https://breakingdefense.com/2019/03/us-loyal-wingman-takes-flight-afrl-kratos-xq-58avalkyrie/ 